\title{
Viability of Parhyale hawaiensis for chronic ecotoxity testing: the case study of diflubenzuron
}

Patrícia Kushima Assano*, Otávio Jürgensen Diehl, Amanda dos Santos, Francine Inforçato Vacchi, Rhaul de Oliveira, Gisela de Aragão Umbuzeiro

\begin{abstract}
Parhyale hawaiensis is an marine amphipod and attractive model organism for ecotoxicology. The insect growth regulator diflubenzuron (DFB) is a chitin syntheis inhibitor insecticide with have pronounced toxic effects on non-target organisms, especially insects and crustaceans. This study aimed to evaluate the toxicity assessment of diflubenzuron using a miniaturized approach for chronic test with Parhyale hawaiensis, previousily standarized in our reasearch group.
\end{abstract}

Key words: Crustacean, toxicity test, chronic exposure.

\section{Introduction}

DFB is a chitin synthesis-inhibiting benzoylurea pesticide that specifically targets the arthropod moulting process. Because of the similarities between insect and crustacean cuticles, worth concern about potential adverse effects of DFB on crustaceans. Parhyale hawaiensis has become a model in marine research, but its potential for chronic toxicity assessment still unclear ${ }^{1}$. The aim of this study was to evaluate both chronic effects of DFB on $P$. hawaiensis comparing the obtained data with acute toxicity responses and other studied species using a Species Sensitivity Distribution analysis (SSD).

\section{Results and Discussion}

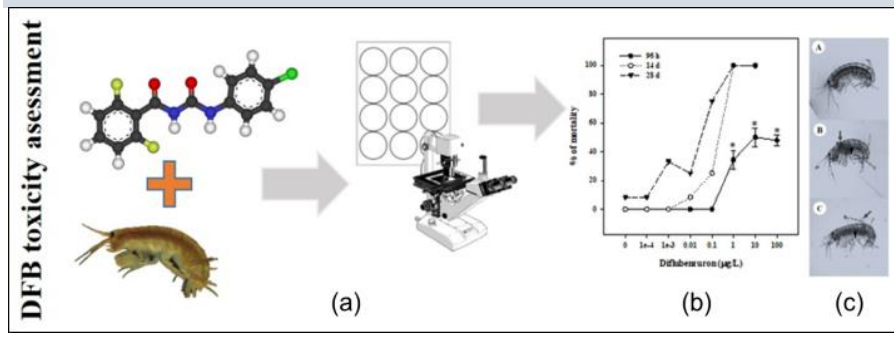

Figure 1. Test scheme for chronic toxicity assessment (a). DFB concentration dose response curves for mortality (b). Visual morphological deformities (c).

For acute toxicity assessment, three independent experiments were performed at DFB concentrations of 0 , $0.01,0.1,1,10$, and $100 \mu \mathrm{g} \mathrm{L}^{-1}$ plus a solvent control, following the methodology described by Artal et al. $(2018)^{1}$. For chronic toxicity assessment (Figure 1), 12 organisms per treatment at ages $<7$ days $^{1}$ were distributed in 12-well microplates containing $4 \mathrm{~mL}$ of reconstituted seawater ( $30 \pm 2$ salinity, Red Sea Salt ${ }^{\circledR} ; \mathrm{pH}$ $8 \pm 1$; water quality of $6 \pm 2 \mathrm{mg} \mathrm{L}^{-1}$ dissolved oxygen); with abiotic conditions of $24 \pm 2{ }^{\circ} \mathrm{C}$; photoperiod $12 \mathrm{~h}$ light; and one crushed coral. Three times per week, organisms were fed $\mathrm{fe}^{2,4}$ with approximately $3 \mathrm{mg}$ of a commercial sinking fish food pellet. Alive neonates were placed in a new 12-well microplate with fresh exposure solution. The DFB concentrations used were: $0,0.0001$, $0.001,0.01,0.1,1$, and $10 \mu \mathrm{g} \mathrm{L}^{-1}$ plus a solvent control; Duration lasted for 28 days. Organisms were monitored daily for moults and mortality. Every week a photo was taken to measure the cephalothorax length $(\mathrm{mm})$ and enable to study the growth rate.

Regarding the growth rate none of the treatment groups were significantly different from the controls (by Kruskal-
Wallis, $\mathrm{p}<0.05$ ). For mortality the $28 \mathrm{~d}$-LC50 $=1.2 \times 10^{-2}$ $\mu \mathrm{g} \mathrm{L}-1$ were obtained, a value $\sim 250$ times lower the $96 \mathrm{~h}$ LC50 = 3.1 $\mu \mathrm{g} \mathrm{L-}{ }^{1}$, for acute toxicity test (Table 1). It was possible to note sub lethal effects of diflubenzuron on morphological features of $P$. hawaiensis after 28 days of exposure, with EC50-28d = $1.3 \times 10^{-2} \mu \mathrm{g} \mathrm{L-1}$.

Table 1. Lethal concentrations $\left(\mu \mathrm{g} \mathrm{L}^{-1}\right)$ of DFB on $P$. hawaiensis (mean values \pm standard deviation).

\begin{tabular}{cccc}
\hline Time & LC10 & LC20 & LC50 \\
\hline $\begin{array}{c}\text { Acute } \\
96 \mathrm{~h}\end{array}$ & $1.4 \times 10^{-1}\left(7.0 \times 10^{-2}\right)$ & $1.0\left(3.6 \times 10^{-1}\right)$ & $3.1(1.1)$ \\
Chronic & & & \\
$14 \mathrm{~d}$ & $3.2 \times 10^{-2}\left(1.8 \times 10^{-2}\right)$ & $5.5 \times 10^{-2}(0.0245)$ & $1.4 \times 10^{-1}\left(5.2 \times 10^{-2}\right)$ \\
$28 \mathrm{~d}$ & $2.5 \times 10^{-4}\left(2.7 \times 10^{-4}\right)$ & $1.0 \times 10^{-3}\left(8.5 \times 10^{-4}\right)$ & $1.2 \times 10^{-2}\left(6.9 \times 10^{-3}\right)$ \\
\hline
\end{tabular}

SSD showed $P$. hawaiensis as the most sensitivity specie to diflubenzuron (Figure 2).

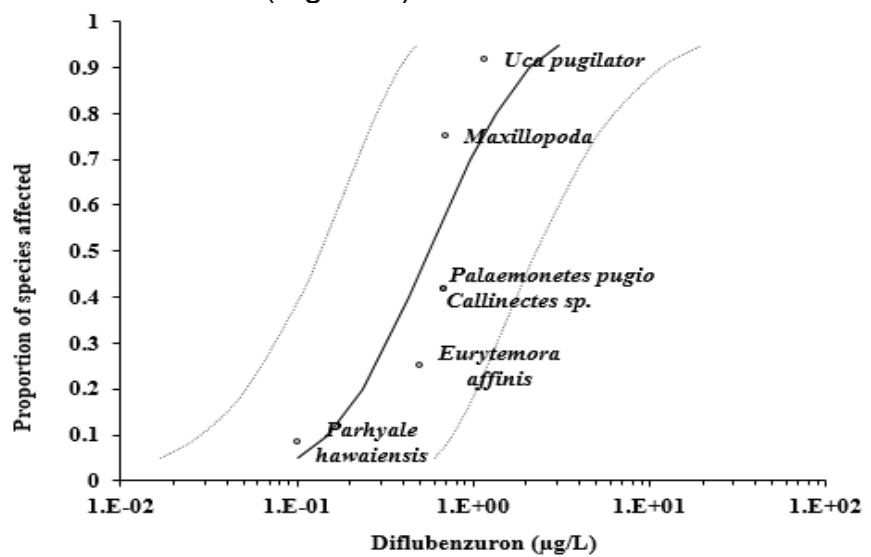

Figure 2. SSD analysis for DFB chronic toxicity data.

\section{Conclusions}

Thus, diflubenzuron is extremely toxic to $P$. hawaiensis. No significantly effects on growth rate were observed, but effects on survival and morphological features of $P$. hawaiensis after 28 days of exposure were observed.

\section{Acknowledgement}

CNPQ, PIBIC, Mariana C. Artal, Gabriel R. Magalhães, Ádria C. Oliveira, Aline A. Godoy, Anjaina F. Albuquerque, Josiane A. S. Vendemiatti and Carina Azevedo.

${ }^{1}$ Artal, M. C., dos Santos, A., Henry, T. B., \& de Aragão Umbuzeiro, G. (2018). Development of an acute toxicity test with the tropical marine amphipod Parhyale hawaiensis. Ecotoxicology, 27(2), 103-108. 